\section{Deutsche Hausärzte sind richtig gefrustet}

Der Frust unter deutschen Hausärzten ist groß: Nach der Zufriedenheit mit ihrer beruflichen Situation gefragt geben $60 \%$ an, etwas oder sehr unzufrieden zu sein. In Norwegen sind es hingegen nur 10\%. Befragt wurden 10.320 Ärzte in elf verschiedenen Ländern. Koch K et al, Dtsch Arztebl Int 2011, 108:255

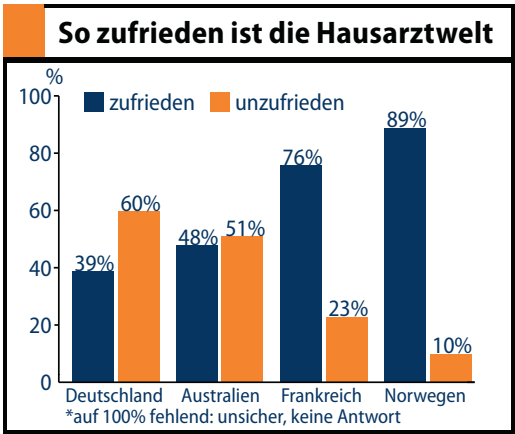

\section{Johanniskraut nicht gleich Johanniskraut}

Echt muss es sein, das Johanniskraut, aus dem der Extrakt gegen Depressionen gewonnen wird. Bei Präparaten aus Drogeriemärkten sei dies jedoch nicht immer der Fall, warnt die Bundesvereinigung Deutscher Apothekerverbände (ABDA). In einer Stichprobe, bei der freiverkäufliche mit apothekenpflichtigen Präparaten verglichen wurden, entdeckten die Forscher eine andere Johanniskrautart aus China. Pressemitteilung ABDA 28.04.11

\section{Schlappe Schilddrüse macht farbenblind}

Erst eine schlappe Schilddrüse, dann auch noch farbenblind? Eine Hypothyreose führt aktuellen Forschungen zur Folge zu Veränderungen der Zapfen der Retina, die eine Grünblindheit zur Folge haben. Zum Glück komme eine solch schwere Hypothyreose, wie in diesem Tierexperiment in der Klinik jedoch nur selten vor, so die Forscher. Glaschke A et al, J Neurosci 2011, 31:4844

\section{Eine Woche Pause reicht}

Nach Infarkt nicht zu lange ausruhen
Nach dem überstandenen Herzinfarkt erst einmal auf die Couch statt ins Sportstudio? Wer diese Art der Rehabilitation bevorzugt, muss umdenken!

Ein gesundes Herz braucht Arbeit, um gesund zu bleiben. Ein krankes, um gesund zu werden, aber auch! Kanadische Forscher haben die Empfehlung der körperlichen Schonung nach einem Herzinfarkt kritisch unter die Lupe genommen und kommen zu einem interessanten Ergebnis: Bei der Auswertung von 1029 Studien zu diesem Thema stellten sie fest, dass sich die Ejektionsfraktion umso langsamer besserte, je später mit körperlicher Betätigung begonnen wurde.

Auch umgekehrt traf dieser Zusammenhang zu: Die Herzfunktion erholte sich umso schneller, je früher die Patienten wieder körperlich aktiv wurden. Dabei liefert die Studie auch konkrete Zahlen: Mit jeder Woche, die gewartet wurde, benötigten die Patienten einen Monat mehr Training, um das gleiche Ausmaß an Remodeling des linken Ventrikels zu erreichen. Außerdem wichtig: Nicht zu früh aufhören! Mindestens sechs Monate sollte trainiert werden, damit es den Herzinfarktpatienten wirklich etwas nützt. Haykowsky et al, Trails 2011, 12:92

\section{Bloß nicht trocknen, seifen, weiten Intimer Putzfimmel lockt HIV}

Wenn Frauen meinen, sie müssten ihre Vagina mit Seife und Reinigungsmitteln bearbeiten, kann das nicht nur zu bakteriellen Vaginosen führen!

Eine durch vaginale Reinigungspraktiken gestörte Vaginalflora bedeutet auch ein erhöhtes HIV-Infektionsrisiko. Dies ergab eine Metaanalyse der Daten von 14.874 Frauen aus 13 prospektiven Kohortenstudien. Von diesen Frauen infizierten sich 791 im Verlauf von 21.218 Frauenjahren mit HIV. Als signifikante und von anderen Faktoren wie Alter oder Zahl der Geschlechtspart- ner unabhängige Risikofaktoren für eine HIV-Infektion entpuppten sich intravaginale Anwendungen von Stoff oder Papier, Einführen von Produkten zum Trocknen oder Weiten der Vagina und intravaginale Reinigung mit Seife.

Wie zu erwarten, war das Reinigen der Vagina mit Seife außerdem selbst bei Frauen mit zu Beginn intakter Vaginalflora mit dem Risiko der Entwicklung einer Mischflora oder einer bakteriellen Vaginose verbunden. Diese waren aber beide wiederum ein Risikofaktor für eine HIV-Infektion. 